POS $\quad \begin{aligned} & \text { PROCEEDINGS } \\ & \text { OF SCIENCE }\end{aligned}$

\title{
Search for stop and sbottom pair production at the LHC using the CMS detector
}

\section{Loukas Gouskos for the CMS Collaboration*}

University of Athens, Greece

E-mail: Loukas.Gouskosecern.ch

In this talk, the latest results from CMS on direct stop or sbottom pair production are reviewed. We present searches preformed for different stop and sbottom decay modes using up to 20/fb of data from the $8 \mathrm{TeV}$ LHC run of 2012.

XXI International Workshop on Deep-Inelastic Scattering and Related Subject -DIS2013, 22-26 April 2013

Marseilles, France

${ }^{*}$ Speaker. 


\section{Introduction}

Despite the success of the theory of standard model (SM) to describe extremely successfully the particle physics phenomena, there are still extensions needed to describe physics at higher energies. Supersymmetry (SUSY) is considered as one of the most appealing extensions, since it provides a natural mechanism to cancel the quadratic divergences appearing in the calculated Higgs mass caused by states of much higher (e.g. Planck) scale, known as hierarchy problem. In order to have a natural solution to the hierarchy problem, SUSY requires light third generation quarks and gluinos [1],[2]. In addition, SUSY leads to unification of the gauge couplings. If R-parity is conserved, the lightest supersymmetric particle (LSP) is stable and provides a good dark matter candidate.

Third generation (top and bottom) squarks can be produced either by decays of heavier SUSY particles such as gluino (gluino mediated production) or directly in pairs (direct production). Figure 1 shows example topologies for indirect (left) and direct (middle and right) stop production mechanisms for R-parity conserving SUSY. The existence of two undetected LSPs leads to a final state with large missing energy. In this document, preliminary results of searches in the final state with a single lepton are presented. These searches are based on data collected by the CMS detector [3] at the LHC in the $2012 \mathrm{pp}$ collision at a center of mass energy $\sqrt{8} \mathrm{TeV}$, corresponding to an integrated luminosity up to $19.4 / \mathrm{fb}$. In the absence of significant excess above the SM expectation, exclusion limits are derived in the context of the simplified model spectra (SMS).
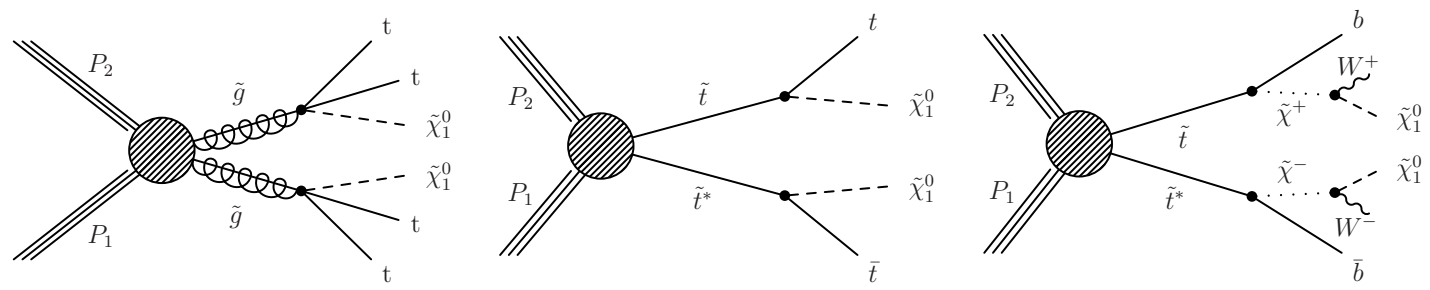

Figure 1: Example of the two top squark production mechanisms. The gluino induced stop production (left) and the two decay channels for direct stop production (middle and right).

\section{Search for gluino induced top squark production in the single lepton final state}

This section presents the results of the search for gluino induced stop production, based on an integrated luminosity of $19.4 / \mathrm{fb}$ data. Due to the four W bosons in the final state of this signature, the single lepton final state has the largest branching fraction $(\sim 40 \%)$. The search requires an isolated muon or electron with $p_{T}>20 \mathrm{GeV}$ [4]. We require six or more jets with $p_{T}>40 \mathrm{GeV}$, at least two of which are identified as b-quark jets by the Combined Secondary Vertex medium working point (CSVM) b-tagging algorithm. Two independent methods have been developed to estimate the background.

The first, is using the azimuthal angle between the reconstructed $\mathrm{W}$ boson direction and the charged lepton, $\Delta \phi$ (W,lepton) to suppress mainly the single lepton SM backgrounds (W+jets, $t \bar{t}(1 l))$. The signal region is defined as $\Delta \phi(\mathrm{W}, 1)>1$ and the dominant background is $t \bar{t}(2 l))$ where 


\begin{tabular}{|c|c|c|c|c|c|}
\hline & & $\mathrm{S}_{T}^{l e p}[\mathrm{GeV}]$ & control reg. data & prediction & observation \\
\hline \multirow{6}{*}{$\begin{array}{l}\pi_{0} \\
z^{0}\end{array}$} & \multirow{3}{*}{$\stackrel{\tilde{0}}{\stackrel{\Xi}{\Sigma}}$} & {$[250,350]$} & 141 & $6.00 \pm 2.40(2.23)$ & 9 \\
\hline & & {$[350,450]$} & 24 & $1.37 \pm 1.19(1.12)$ & 2 \\
\hline & & $>450$ & 9 & $0.0 \pm 0.66(0.66)$ & 0 \\
\hline & \multirow{3}{*}{$\begin{array}{r}\ddot{U} \\
\frac{\vec{U}}{\Delta I}\end{array}$} & {$[250,350]$} & 112 & $3.83 \pm 1.84(1.75)$ & 9 \\
\hline & & {$[350,450]$} & 28 & $2.74 \pm 2.02(1.86)$ & 2 \\
\hline & & $>450$ & 9 & $0.0 \pm 0.42(0.42)$ & 0 \\
\hline \multirow{6}{*}{$\begin{array}{l}\hat{\wedge} \\
\sum^{0}\end{array}$} & \multirow{3}{*}{$\stackrel{\tilde{O}}{0}^{\tilde{z}}$} & {$[250,350]$} & 28 & $1.92 \pm 0.95(0.84)$ & 0 \\
\hline & & {$[350,450]$} & 13 & $0.57 \pm 0.58(0.52)$ & 0 \\
\hline & & $>450$ & 2 & $0.0 \pm 0.22(0.22)$ & 0 \\
\hline & \multirow{3}{*}{$\frac{\dot{U}}{\frac{0}{\Delta I}}$} & {$[250,350]$} & 45 & $1.89 \pm 1.03(0.94)$ & 4 \\
\hline & & {$[350,450]$} & 7 & $0.85 \pm 0.80(0.70)$ & 0 \\
\hline & & $>450$ & 0 & $0.0 \pm 0.08(0.08)$ & 0 \\
\hline
\end{tabular}

Table 1: Event yields in $19.4 \mathrm{fb}^{-1}$ of data with $N_{\text {jet }} \geq 6$ : the columns list the numbers of events observed in the control region, while for the signal region both the numbers of events expected and the numbers of events observed are listed. The uncertainty reflects the total uncertainty, while the number in parenthesis the statistical uncertainty stemming from the number of events in the control regions.

the second lepton is not reconstructed or out of the detector acceptance. The control region is defined as $\Delta \phi(\mathrm{W}, 1)<1$. The search is performed in different regions of the quantity, $\mathrm{S}_{T}^{\text {lep }}$, defined as the scalar sum of $\mathrm{ME}_{T}$ and charged lepton $p_{T} . \mathrm{S}_{T}^{l e p}$ is a measure of the leptonic energy scale of the event, with SUSY signals expected to yield events at large $S_{T}^{l e p}$, where the contribution from SM processes is significantly reduced. To increase the sensitivity, the search is performed in different regions of the b-quark multiplicities $\left(\mathrm{N}_{b}\right)$. Figure 2 (left) shows an example distribution of the $\Delta \phi(\mathrm{W}, \mathrm{l})$ variable for the main SM processes and three competitive SUSY models. The background estimation uses a translation factor from the control to signal region, $\mathrm{R}_{C S}$ :

$$
R_{C S}=\frac{N_{\text {signal }}}{N_{\text {control }}}=\frac{\text { Number of events with } \Delta \phi(W, l)>1}{\text { Number of events with } \Delta \phi(W, l)<1} .
$$

The value of $\mathrm{R}_{C S}$ is roughly independent of the b-quark multiplicity[4]. This property is the primary motivation for the analysis strategy: $\mathrm{R}_{C S}$ can be measured in data in the background dominated sample with $\mathrm{N}_{b}=1$. To account residual differences of $\mathrm{R}_{C S}$ with $\mathrm{N}_{b}$ we also introduce a correction factor, $\kappa_{R C}$ from simulation. The SM estimation in the signal region is obtained by:

$$
N_{\text {signal }}\left(N_{b}=2, \geq 3\right)=R_{C S}\left(N_{b}=1\right) \cdot \kappa_{C S}\left(N_{b}=2, \geq 3\right) \cdot N_{\text {control }}\left(N_{b}=2, \geq 3\right) .
$$

Using Monte Carlo simulated samples, it was found that the QCD background is much smaller compared to the other SM processes, totally negligible in the muon channel. In the electron channel, the estimation of the QCD contribution is based on a method developed here [5],[6] using data to large extend. The QCD contamination is less than 5\% of the total data and negligible in $\Delta \phi(\mathrm{W}, \mathrm{l})>1$. Thus the number of $\mathrm{QCD}$ events is subtracted only from the control region.

The ingredients of the analysis are tested in a background dominated control sample with selection identical to the search sample but with lower jet multiplicity $\left(3 \leq N_{\text {jets }} \leq 5\right)$. The results show good agreement between the observed data and the SM expectation [4]. Table 1 shows the results of the search for each region. No evidence of gluino induced stop production is observed. 
The second method, is called the Lepton Spectrum method and uses the lepton transverse momentum distribution in data to predict the missing energy distribution for single lepton processes. Correction factors from simulation are introduced to correct for residual differences. In contrast to the first method, here the dominant background is $t \bar{t}(1 l)$. The remaining backgrounds are estimated with additional background estimation methods. The search is performed in different regions of $\mathrm{ME}_{T}$ and $\mathrm{H}_{T}$. Figure 2 (right) shows the observed $\mathrm{ME}_{T}$ distributions in data compared to the predicted distributions in the combined electron and muon channels, for events with $\mathrm{N}_{\text {jets }} \geq 6$ and $\mathrm{H}_{T}>500 \mathrm{GeV}$. No evidence of gluino induced stop production is observed [4].
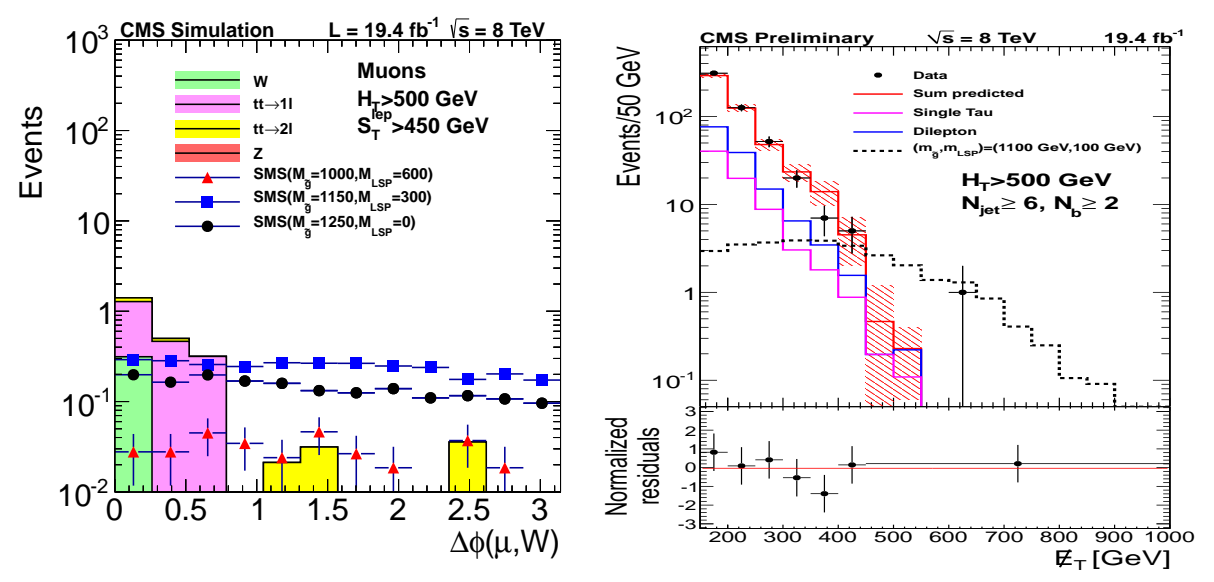

Figure 2: Example distribution of the $\Delta \phi(\mathrm{W}, 1)$ variable for the main SM processes and three competitive SUSY models for the $\Delta \phi$ method (left). Observed $\mathrm{ME}_{T}$ distributions in data compared to the predicted distributions in the combined electron and muon channels, for events with $\mathrm{N}_{\text {jets }} \geq 6$ and $\mathrm{H}_{T}>500 \mathrm{GeV}$ for the Lepton Spectrum Method (right).

The observed data are compatible with the SM estimations in both methods and the results are interpreted using the gluino induced stop production model. The model is parameterized by the masses of the gluino and the $\tilde{\chi}_{0}^{1}$. Figure 3 (left) shows the excluded parameter space for both methods. The search probes gluino masses $\sim 1280 \mathrm{GeV}$ for an $\tilde{\chi}_{0}^{1}$ mass of $\sim 570 \mathrm{GeV}$. Figure 3 (right) compares the limits for different final states in the same topology.

\section{Search for direct top squark production in the single lepton final state}

This section presents the results of a dedicated search for direct production of stop pairs, based on an integrated luminosity of $9.7 / \mathrm{fb}$. The presence of the two $\mathrm{W}$ bosons in the final state leads to significant branching fraction for the single lepton topology. The search requires an isolated muon or electron with $p_{T}>30 \mathrm{GeV}$ and at least four jets with $p_{T}>40 \mathrm{GeV}$ [7]. At least one of these jets should be identified as b-quark by the CSVM b-tagging algorithm. The dominant background is $t \bar{t}(1 l)$ and $t \bar{t}(2 l)$. To further suppress the $t \bar{t}(2 l)$ background events with an additional isolated charged particle are rejected. The search is performed for different $\mathrm{ME}_{T}$ and $\mathrm{M}_{T}\left(1, \mathrm{ME}_{T}\right)$ region to account for the unknown mass scale of SUSY.

The SM backgrounds in the various signal regions are estimated by extrapolation factors from simulation. The expectation from MC is normalized to the data in the $\mathrm{M}_{T}$ peak region $(50 \rightarrow 80$ 

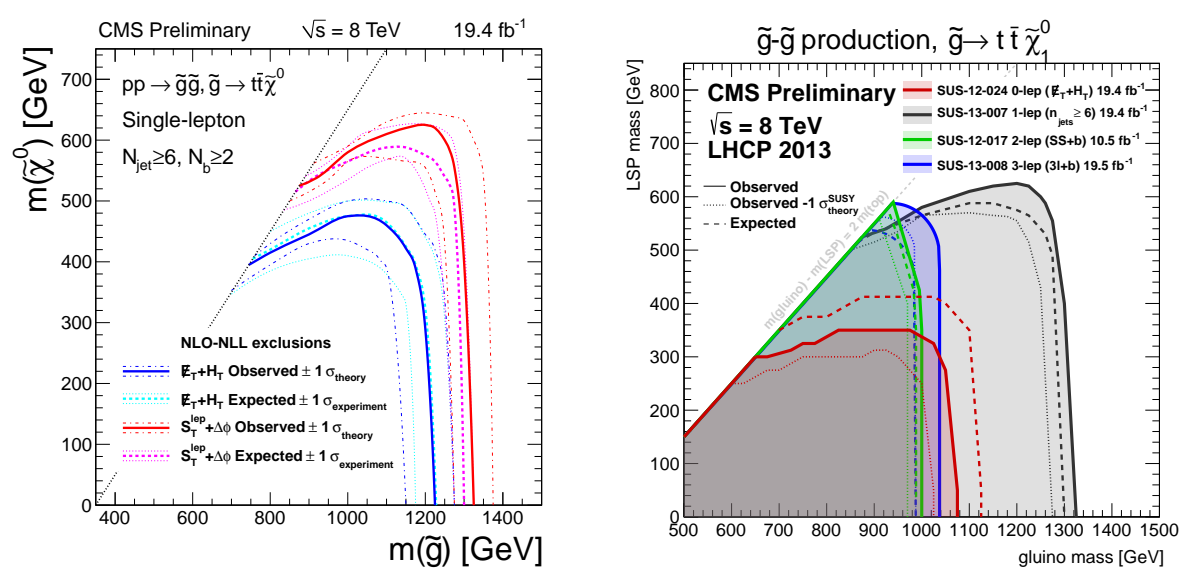

Figure 3: Excluded parameter space in gluino induced stop production for both methods in the single lepton final state (left) and comparison of the limits for different final states in the same topology (right).

$\mathrm{GeV}$ ) and then extrapolated to the large $\mathrm{M}_{T}$ region. By this method, systematic uncertainties related to the luminosity calculation and $t \bar{t}$ cross section, cancel to large extend. The extrapolation factors are validated in data control regions and where necessary corrected. Four control regions, based on different selection criteria, are defined to validate the simulation of the dominant SM processes (W+jets, $t \bar{t}(1 l)$ and $t \bar{t}(2 l)$ ) and extract the correction factors by comparing the MC expectation and the observed data. These control regions are used to derive systematic uncertainties in the background prediction for each process. The dominant systematic uncertainty is due to the limited statistical precision in the data control samples used for these tests.

The data observed in the different search regions and the SM expectation show no evidence of supersymmetric signal. Figure 4 (left) shows the $\mathrm{ME}_{T}$ distribution in data compared to the $\mathrm{SM}$ expectations. Two example signal models are used. The results are interpreted in the context of direct top squark pair production. Upper limits are placed on the signal production cross section in the two dimensional parameter space, using that search region that provides the best expected limit. The search probes top squarks with masses up to $\sim 430 \mathrm{GeV}$ and LSP masses smaller than $\sim 120$ $\mathrm{GeV}$, depending on the scenario. Figure 4 (right) shows as an example the limit in the $\tilde{t} \rightarrow t \tilde{\chi}_{0}^{1}$ scenario.

\section{Summary}

Supersymmetry is an appealing extension to the standard model theory and may provide a natural solution to the hierarchy problem by introducing third generation squarks with masses at the order of several hundred $\mathrm{GeV}$. We presented results for gluino induced top squark production and direct top squark pair production in final states with a single lepton. No evidence of supersymmetric signal is observed. The results used to constraint even further the available parameter space.

\section{Acknowledgments}

Co-funded by the European Union (European Social Fund âĂŞ ESF) and Greek national funds 

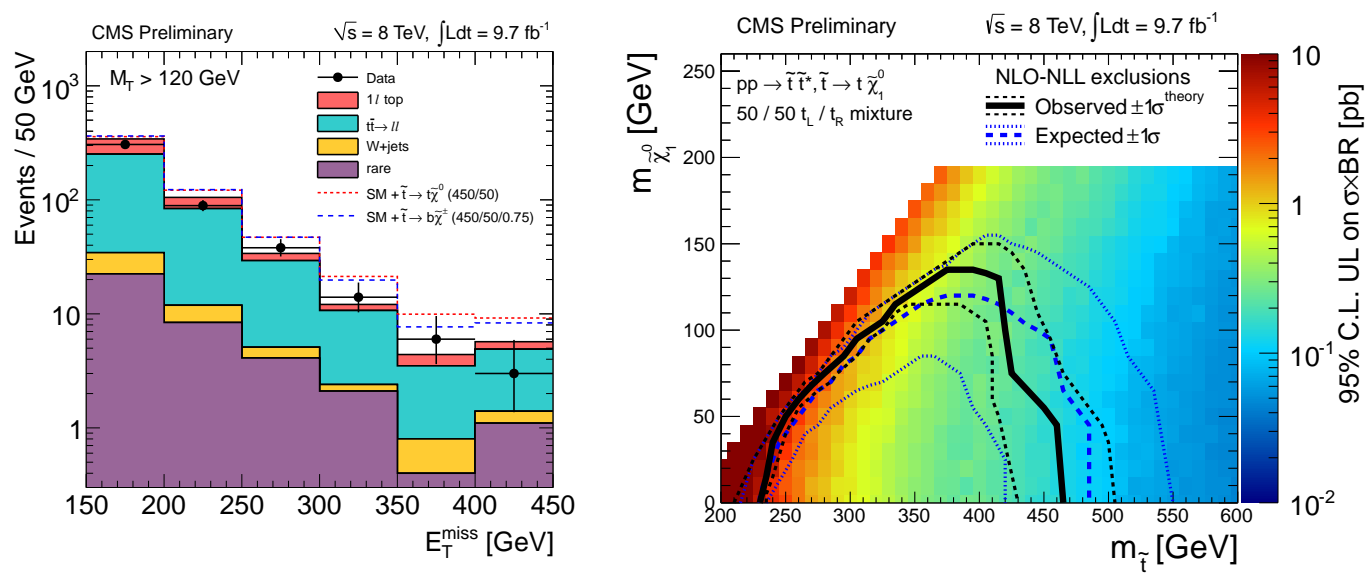

Figure 4: $\mathrm{ME}_{T}$ distribution in data compared to the $\mathrm{SM}$ expectations (left) and Interpretation of the results in the $\tilde{t} \rightarrow t \tilde{\chi}_{0}^{1}$ scenario (right).

through the Operational Program "Education and Lifelong Learning" of the National Strategic Reference Framework (NSRF).

\section{References}

[1] S. Dimopoulos et al., Naturalness constraints in supersymmetric theories with nonuniversal soft terms, Phys.Lett. B , 357, (573) [hep-ph/9507282]

[2] N. Sakai, Naturalness in Supersymmetric Guts, Z. Phys. C, 11 (153)

[3] S. Chatrchyan, et al. (CMS Collaboration), The CMS experiment at the CERN LHC, JINST 03 (2008) S08004

[4] S. Chatrchyan, et al. (CMS Collaboration), Search for supersymmetry in pp collisions at $\sqrt{s}=8 \mathrm{TeV}$ in events with a single lepton, multiple jets and b-tags, CMS-SUS-13-007 (2013)

[5] S. Chatrchyan, et al. (CMS collaboration), Measurement of the Polarization of W Bosons with Large Transverse Momenta in W+Jets Events at the LHC, Phys. Rev. Lett., 107 (2011) 021802

[6] S. Chatrchyan, et al. (CMS Collaboration), Eur. Phys. J. C, 73, (2013) 2404

[7] S. Chatrchyan, et al. (CMS Collaboration), Search for direct top squark pair production in events with a single isolated lepton, jets and missing transverse energy at $\sqrt{s}=8 \mathrm{TeV}$, CMS-SUS-12-023 (2012) 